\title{
A 74-Year-Old Woman With Dysphagia: What Is Your Diagnosis by Endoscopic and Barium Swallow Finding?
}

Jeong Woo Lee, MD, Joong Goo Kwon, MD*, Eun Young Kim, MD, Jin Tae Jung, MD, Jimin Han, MD and Ho Gak Kim, MD

Department of Internal Medicine, Catholic University of Daegu School of Medicine, Daegu, Korea

A 74-year-old woman visited the department of gastroenterology by 4 months of dysphagia. Dysphagia was intermittent, nonprogressive and occurred with solid foods. The patient underwent an upper gastrointestinal endoscopy, which showed a clearly demarcated smooth concentric narrowing at 2 $\mathrm{cm}$ above the squamocolumnar junction (Fig. 1A). A barium swallow showed a focal smooth narrowing in the distal esophagus, with openings of variable diameter during the course of examination (Fig. 1B and 1C). What is your diagnosis by endoscopy and barium swallow finding? Considering a possibility of a muscular ring in the distal esophagus, endoscopic ultrasound examination with conventional radial echoendoscopy was performed. Focal thickening of inner circular muscle layer at $2 \mathrm{~cm}$ above the squamocolumnar junction was noted. Esophageal manometry was performed by standard techniques using a water-perfused catheter system (Fig. 2). The lower esophageal sphincter pressure was $63.3 \mathrm{mmHg}$ and relaxed completely with swallows. Postrelaxation high-amplitude contractions of lower esophageal sphincter
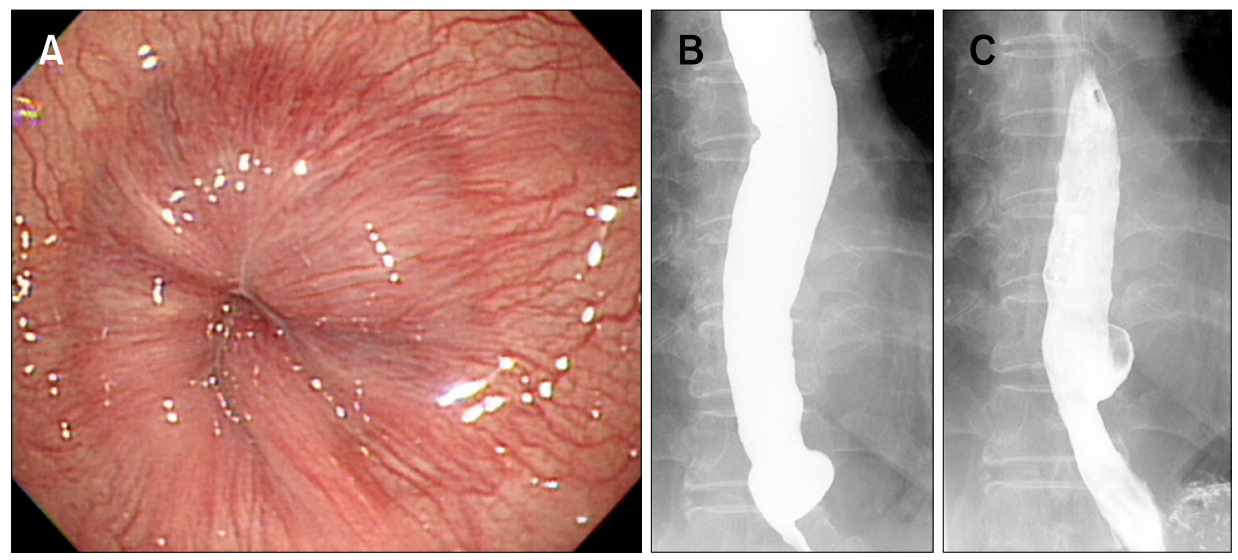

Figure 1. (A) Upper gastrointestinal endoscopic finding shows a concentric ring-like mucosal narrowing just above the squamocolumnar junction. (B) Barium esophagogram shows focal luminal constriction through which a small amount of barium transit into the stomach. (C) The same area of the esophagus showing relative opening of the constriction.

Received: May 31, 2010 Revised: June 13, 2010 Accepted: June 15, 2010

(c) This is an Open Access article distributed under the terms of the Creative Commons Attribution Non-Commercial License (http://creativecommons. org/licenses/by-nc/3.0) which permits unrestricted non-commercial use, distribution, and reproduction in any medium, provided the original work is properly cited.

*Correspondence: Joong Goo Kwon, MD

Department of Internal Medicine, Catholic University of Daegu School of Medicine, 3056-6 Daemyung 4-dong, Nam-gu, Daegu 705-718, Korea

Tel: +82-53-650-4215, Fax: +82-53-628-4005, E-mail: kwonjg@cu.ac.kr

Financial support: None.

Conflicts of interest: None. 


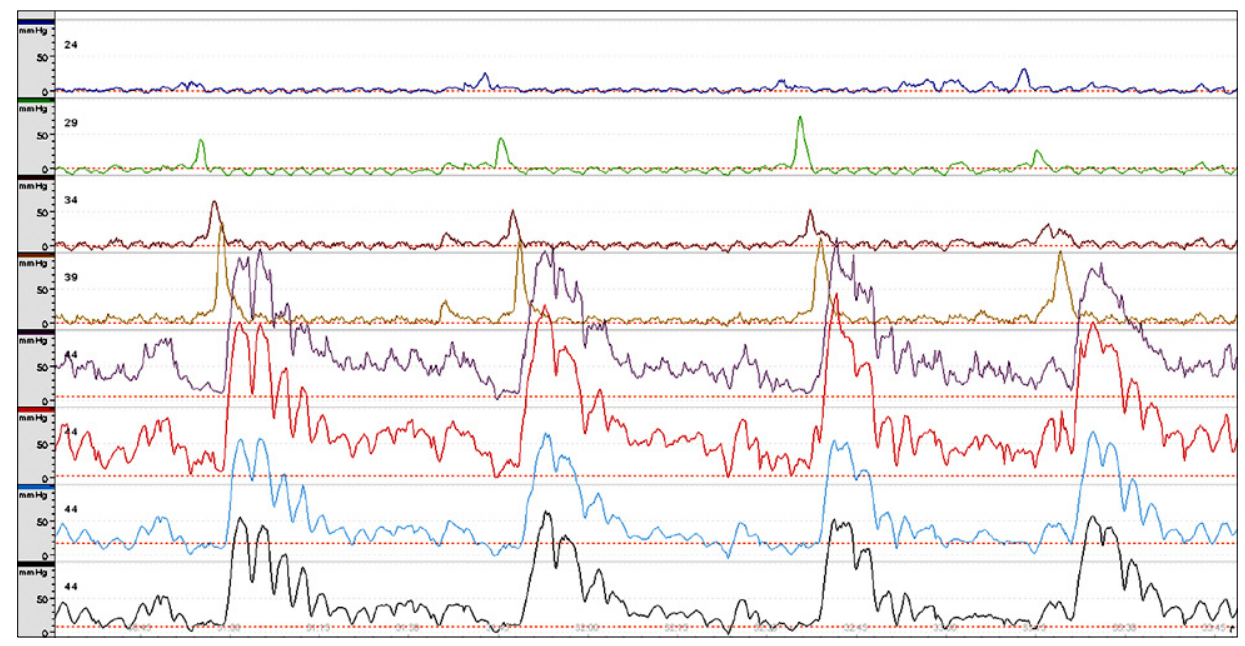

Figure 2. Esophageal manometric findings. Wet swallows evoke normal peristaltic contractions of esophageal body and complete relaxation of lower esophageal sphincter (LES). Postrelaxation high-amplitude contractions of LES are also observed with intermittent multiple-peaked contractions. were observed with intermittent multiple-peaked contractions. Amplitudes of the esophageal contraction waves were normal and propagated well. High-amplitude, long-duration contraction of esophageal body was not observed during 10 wet swallows. She was diagnosed as having a lower esophageal muscular ring and treated with calcium channel blocker. On follow-up after 6 months, she was doing well and complete resolution of dysphagia was noted. Esophageal muscular ring is an uncommon cause of dysphagia. ${ }^{1-3}$ Distinguishing the muscular ring from achalasia and other causes of focal esophageal stenosis is important because of the differences in treatment and outcome. ${ }^{4}$

\section{References}

1. Røhl L, Aksglaede K, Funch-Jensen P, Thommesen P. Esophageal rings and strictures. Manometric characteristics in patients with food impaction. Acta Radiol 2000;41:275-279.

2. Park KC, Kwon JG, You GJ, et al. A case of lower esophageal muscular ring presenting with dysphagia. Korean J Gastroenterol 2003; 42;539-543.

3. Goyal RK, Bauer JL, Spiro HM. The nature and location of lower esophageal ring. N Engl J Med 1971;284:1175-1180.

4. Hirano I, Gilliam J, Goyal RK. Clinical and manometric features of the lower esophageal muscular ring. Am J Gastroenterol 2000; 95:43-49. 